\title{
Ontological Pluralism and Notational Variance
}

\author{
Bruno Whittle
}

July 18, 2017

Ontological pluralism is the view that there are different ways to exist. It is a position with deep roots in the history of philosophy. For example, Aristotle seemed to endorse it when he said that 'there are many senses in which a thing may be said to 'be". ${ }^{1}$ Although the view fell out of favour, there has recently been a resurgence of interest, sparked by defences from Kris McDaniel [2009, 2010a, 2010b] and Jason Turner [2010, 2012]. Indeed, while the position may still have relatively few adherents in quite these terms, the influential Fregean approach to higher-order quantification-according to which this is over 'concepts' rather than objectswould seem to be an instance of it. ${ }^{2}$

In contemporary presentations, the view is stated in terms of fundamental languages. ${ }^{3}$ That is, languages whose expressions 'carve nature at the joints', or whose meanings are natural in the sense of Lewis $[1983,1986]$. Thus stated, it is the claim that such languages have more than one type of quantification, ranging over different domains. For example, $\exists_{a}$ ranging over abstract objects, and $\exists_{c}$ ranging over concrete ones. ${ }^{4}$

\footnotetext{
${ }^{1}$ Metaphysics IV.2. For more on the history of ontological pluralism, see McDaniel [2009] and Caplan [2011].

${ }^{2}$ See Frege $[1891,1892]$. A similar point could be made about many other approaches to highorder quantification, e.g. those of Russell [1908], Prior [1971] and Williamson [2003]. On the relation between ontological pluralism and higher-order quantification, see Turner [2010: 12-13] and Caplan [2011].

${ }^{3}$ See McDaniel [2009] and Turner [2010, 2012]. On fundamental languages more generally, see Sider [2011].

${ }^{4}$ In fact, there is some debate about how exactly the position should be stated: see McDaniel [2009], Turner [2010], Caplan [2011] and Spencer [2012]. For example, it has been suggested that Lewisian naturalness might be replaced by Schafferian fundamentality (see Caplan [2011: 91-93]), or that, in the spirit of Wittgenstein's Tractatus, it be stated without reference to quantification at all, but rather 'sorts' of terms and argument places (see Turner [2010: 10-11]). Most of these con-
} 
There is, however, a worry that one might have about ontological pluralism. This is not a worry that it is false, but rather that it is not substantively different from its supposed rival, (ontological) monism. In particular, one might worry that the languages proposed by the pluralist are mere notational variants of those proposed by the monist. For example, one might fear that the pluralist language $\mathcal{L}_{P}$ with quantifiers $\exists_{a}$ and $\exists_{c}$ is a mere such variant of the monist language $\mathcal{L}_{M}$, with the single quantifier $\exists$ but predicates Abstract and Concrete.

By way of analogy, consider the languages $\mathcal{L}_{\square}$ and $\mathcal{L}_{\diamond}$, identical except that the first contains $\square$, but not $\diamond$, while the second is the opposite. If one came across two people arguing over which of these is a fundamental language, one would presumably not think that this was an important philosophical question that had been too long neglected. Rather, one would think that it is not a substantive question at all. The worry is that $\mathcal{L}_{P}$ stands in the same sort of relation to $\mathcal{L}_{M}$ that $\mathcal{L}_{\square}$ stands in to $\mathcal{L}_{\diamond}$, and thus that the debate between the pluralist and the monist is similarly insubstantive.

However, Turner [2012] has recently given an ingenious response to this concern, employing a principle that he calls 'logical realism.' According to this, if two fundamental languages are notational variants, under a given translation, then this translation must preserve logic. That is, a formula must be a consequence of a given set of formulas iff this is also true of their translations. But, Turner argues, unlike in the case of $\mathcal{L}_{\square}$ and $\mathcal{L}_{\diamond}$, the translation that threatens to show that $\mathcal{L}_{P}$ and $\mathcal{L}_{M}$ are notational variants fails to preserve logic.

The purpose of this paper is to offer a counter-response on behalf of the 'notationalist'. I argue that, properly applied, the principle of logical realism is no threat to the claim that $\mathcal{L}_{P}$ and $\mathcal{L}_{M}$ are notational variants. Indeed, there seems to be every reason to think that they are.

The structure of the paper is as follows. $\$ 1$ contains preliminaries. $\$_{2}$ gives the worry, and $\S_{3}$ Turner's response. $\$_{4}$ argues against this response. An appendix extends the argument to a variety of pluralism not considered in the main text: that according to which the fundamental language is 'sorted'.

siderations are orthogonal to the concerns of this paper. The exception is the 'Tractarian' question: the arguments to follow are in terms of quantification, and it would take work to reframe them in other terms. However, almost all recent discussion of ontological pluralism has similarly assumed that is stated in quantificational terms.

${ }^{5}$ Strictly speaking, Turner uses 'logical realism' for the view that motivates the principle, and (LR) for the principle itself. But for simplicity I use 'logical realism' for the principle. 


\section{Preliminaries}

Throughout the paper I focus on the example from the introduction: the instance of pluralism on which the fundamental language has exactly the quantifiers $\exists_{a}$ and $\exists_{c}$. However, everything that I say carries straightforwardly over to other instances. ${ }^{6}$

More specifically, in the main text I assume that the pluralist proposes a language, $\mathcal{L}_{P}$, that is just like a standard first-order language, except that it has $\exists_{a}$ and $\exists_{c}$ in place of $\exists$. That is, $\mathcal{L}_{P}$ is 'unsorted': with one sort of term and argument place, rather than distinct sorts corresponding to the two quantifiers. However, as I show in the appendix, a version of the argument to follow can also be given in the case where the a sorted language is proposed. The instance of monism under consideration, then, is that on which the fundamental language, $\mathcal{L}_{M}$, is the standard first-order language that is just like $\mathcal{L}_{P}$, except that it contains $\exists$ and unary predicate symbols Abstract and Concrete, in place of $\exists_{a}$ and $\exists_{c}$. Finally, I assume that $\mathcal{L}_{P}$ and $\mathcal{L}_{M}$ are languages with equality.

To discuss the question of notational variance, we need the concept of a translation. Thus, if $\mathcal{L}$ and $\mathcal{L}^{\prime}$ are languages, a translation between $\mathcal{L}$ and $\mathcal{L}^{\prime}$ is a pair $\left\langle t, t^{\prime}\right\rangle$ such that $t$ is a function from formulas of $\mathcal{L}$ to those of $\mathcal{L}^{\prime}$, and $t^{\prime}$ is one in the opposite direction. However, I often abuse notation and use the same symbol for both functions, allowing context to disambiguate.

A paradigm example of notational variants are thus a pair of languages along the lines of $\mathcal{L}_{\square}$ and $\mathcal{L}_{\diamond}$, under the translation that sends $\square \varphi$ to $\neg \diamond \neg t(\varphi)$, and $\diamond \psi$ to $\neg \square \neg t(\psi)$. Similarly, $\mathcal{L}_{\neg \wedge}$ and $\mathcal{L}_{\neg \vee}$, or $\mathcal{L}_{\exists}$ and $\mathcal{L}_{\forall}$. For simplicity, I build into the definition of notational variance the requirement that the languages involved are fundamental, although one could also, if desired, give a more inclusive definition. Thus, I say that $\mathcal{L}$ and $\mathcal{L}^{\prime}$ are notational variants under translation $t$ if: (i) $\mathcal{L}$ and $\mathcal{L}^{\prime}$ are fundamental languages; and (ii) for any formula $\varphi$ of $\mathcal{L}, t(\varphi)$ 'says the same thing' as $\varphi$, and similarly in the other direction. This characterization is of course less than completely precise, but it will suffice for our purposes. I should though say something by way of explanation.

Concerning (i): we need some clause along these lines because we want notational variants to be metaphysically on a par. But (ii) on its own would not establish this: for example, one might hold that there is a translation between $\mathcal{L}_{P}$ and $\mathcal{L}_{M}$ that satisfies (ii), but that nevertheless only one of these languages is fundamental;

\footnotetext{
${ }^{6}$ This includes those in which the fundamental language has infinitely many quantifiers, as long as one is willing to countenance formulas of infinite length.
} 
in which case one would be metaphysically superior, and the debate between the pluralist and the monist would be substantive.

Concerning (ii): which notion of content is at issue? It cannot be an extremely fine-grained notion, requiring a perfect isomorphism between a formula and what it expresses: since that would rule out paradigm examples such as $\mathcal{L}_{\square}$ and $\mathcal{L}_{\diamond}$. On the other hand, nor can it be a very coarse-grained notion, such as that which identifies contents with sets of possible worlds: for we would then get the result that any two mathematical languages that satisfy (i) are notational variants, which is surely unacceptable. What is needed is a middle way: perhaps the sort of notion Frege had in mind when he famously said that 'the direction of $a$ is the same as the direction of $b$ ' has the same content as ' $a$ is parallel to $b$ '. Such a notion is notoriously difficult to make precise. Fortunately, however, we can to a great extent side-step this issue here: since the heart of the matter is whether the translation between $\mathcal{L}_{P}$ and $\mathcal{L}_{M}$ (to be given in \$2) satisfies the principle of logical realism (i.e. preserves logic); and this question can be addressed quite independently of that of how to explicate such a 'medium-grained' notion of content. ${ }^{8}$

Quite generally, if $\mathcal{L}$ and $\mathcal{L}^{\prime}$ are notational variants, then any debate between their proponents would seem to be insubstantive.

\section{The Worry}

Consider the translation $t$ between $\mathcal{L}_{M}$ and $\mathcal{L}_{P}$, defined by induction as follows. Going first from $\mathcal{L}_{P}$ to $\mathcal{L}_{M}$ : if $\varphi$ is a formula of the form $\exists_{a} x \psi$, then $t(\varphi)$ is $\exists x(\mathrm{Ab}-$ $\operatorname{stract}(x) \wedge t(\psi))$; and similarly if $\varphi$ is $\exists_{c} x \psi$, but with Concrete in place of Ab-

\footnotetext{
${ }^{7}$ See [1884: 74-75]. For discussion of this line of thought in Frege, see Hale and Wright [2001].

${ }^{8}$ Turner [2012: 423] gives a definition of notational variance that promises to avoid this issue. This is in terms of theories rather than languages, and (simplifying inessentially) is as follows. Theories $T$ and $T^{\prime}$ are notational variants under $t$ if: (I) the languages of $T$ and $T^{\prime}$ are fundamental; and (II) $\varphi$ is a theorem of $T$ iff $t(\varphi)$ is one of $T^{\prime}$, and similarly in the other direction. The problem is that this definition is inadequate by Turner's own lights, for it is incompatible with logical realism. This can be seen by letting $T=T^{\prime}$ be some theory in a fundamental language such that for some atomic sentence $\alpha=F s_{1} \ldots s_{n}, \neg \alpha$ is a theorem of $T$, but $\alpha$ is logically contingent (i.e. neither logically true nor false). For consider the translation that sends atomic formulas $\varphi$ of the form $F r_{1} \ldots r_{n}$ to $\varphi \wedge \neg \alpha$; that is the identity on other atomic formulas; and that handles non-atomic formulas in the obvious way. This satisfies (II), but does not preserve logic: because the logically contingent $\alpha$ is sent to the contradiction $\alpha \wedge \neg \alpha$. There does not seem to be any way, therefore, of ultimately avoiding the difficult questions about content raised in the text. I should note, however, that this issue with Turner's definition in no way undermines his argument, since he could just as well use the characterization that I have given.
} 
stract; formulas of other forms are handled in the obvious way. ${ }^{9}$ Going in the opposite direction: if $\varphi$ is $\exists x \psi$, then $t(\varphi)$ is $\exists_{a} x t(\psi) \vee \exists_{c} x t(\psi) ; t(\operatorname{Abstract}(s))$ is $\exists_{a} x x=s$; and $t$ (Concrete $\left.(s)\right)$ is $\exists_{c} x x=s$. Formulas of other forms are handled as before. Thus, for example, $\exists_{a} x \operatorname{Set}(x)$ is translated as $\exists x(\operatorname{Abstract}(x) \wedge \operatorname{Set}(x))$, while $\exists x x=$ harry is translated as $\exists_{a} x x=$ harry $\vee \exists_{c} x x=$ harry.

The problem is that we seem to be dealing here with translations that say the same thing as the formulas translated-and with equal metaphysical transparency. Just as in the case of $\mathcal{L}_{\square}$ and $\mathcal{L}_{\diamond}$, for example. But if that is right, then $\mathcal{L}_{P}$ and $\mathcal{L}_{M}$ are notational variants, and the debate between the pluralist and the monist would seem to be insubstantive.

\section{Logical Realism}

Turner has a response, however, which uses the following principle (see [2012]).

(LR) If $\mathcal{L}$ and $\mathcal{L}^{\prime}$ are notational variants under $t$, then $t$ preserves logic.

That is, for any set $\Gamma \cup\{\varphi\}$ of formulas of $\mathcal{L}, \Gamma \vDash \varphi$ iff $t(\Gamma) \vDash t(\varphi)$; and similarly for any set $\Gamma \cup\{\varphi\}$ of formulas of $\mathcal{L}^{\prime}{ }^{10}$

This principle is supported by the following, apparently very plausible, line of thought (see Turner [2012: 426-27]). If $\Gamma \vDash \varphi$, then this corresponds to an important metaphysical relation between the contents of these formulas-it is not a mere accident of notation. But then this relation should be captured by any metaphysically perspicuous expression of these contents. However, if $\mathcal{L}$ and $\mathcal{L}^{\prime}$ really are notational variants under $t$, then the members of $t(\Gamma) \cup\{t(\varphi)\}$ offer just such an expression, and so we have $t(\Gamma) \vDash t(\varphi)$. That is, if $\Gamma \vDash \varphi$, then $t(\Gamma) \vDash t(\varphi)$. Similarly for the converse: giving (LR).

Armed with this principle, however, Turner has an answer to the worry of $\$ 2$. He points out that the translation $t$ between $\mathcal{L}_{P}$ and $\mathcal{L}_{M}$ does not seem to preserve logic. For consider the following sentence $\alpha_{M}$ of $\mathcal{L}_{M}: \forall x(\operatorname{Abstract}(x) \vee$ Concrete $(x)) .{ }^{11}$ This does not seem to be a logical truth. However its translation does seem to be one. For this (simplifying slightly) is

$$
\alpha_{P}: \forall_{a} x\left(\exists_{a} y y=x \vee \exists_{c} y y=x\right) \wedge \forall_{c} x\left(\exists_{a} y y=x \vee \exists_{c} y y=x\right),
$$

\footnotetext{
${ }^{9}$ I.e. $t$ sends atomic formulas to themselves; $t(\neg \psi)=\neg t(\psi)$; and $t(\psi \wedge \chi)=t(\psi) \wedge t(\chi)$.

${ }^{10}$ Here $\Gamma \vDash \varphi$ means that $\varphi$ is a logical consequence of $\Gamma$, and $t(\Gamma)=\{t(\psi): \psi \in \Gamma\}$.

${ }^{11}$ Universal quantifiers are defined in terms of existential ones in the usual way.
} 
which appears to be a logical truth in virtue of the fact that $\forall_{a} x \exists_{a} y y=x$ and ${ }_{c} x \exists_{c} y y=x$ are. ${ }^{12}$

Perhaps, then, we are not dealing with notational variants after all? ${ }^{13}$

\section{Notational Variance Even So}

One way of trying to counter this response would be to insist that either Abstract or Concrete be defined in terms of the other: for example, that Abstract be defined as $\neg$ Concrete. If such a definition was permitted, then $\alpha_{M}$ would be a logical truth (a quantified instance of the law of excluded middle), and we would no longer have a violation of (LR).

But there seem to be a number of drawbacks with this idea. For one thing, to give such a definition is to treat one of Abstract and Concrete as more fundamental than the other. But it is at least plausible that the properties of being abstract and concrete are equally fundamental. For another, this idea (at least on the most obvious implementations) requires that nothing can be within the range of more than one of the pluralist's quantifiers (see Turner [2012: 428-29]). Perhaps in the case of $\exists_{a}$ and $\exists_{c}$ this is uncontentious. But there are other versions of pluralism where it is very far from so: e.g. that with a quantifier $\exists_{p}$ over the physical, and another $\exists_{m}$ over the mental. Thus, this way of trying to counter Turner's response seems unsatisfactory.

The strategy that I pursue is quite different. The basic idea is this. We only get Turner's result-that $\alpha_{P}$ is a logical truth, while $\alpha_{M}$ is not-if we assume that $\exists_{a}$ and $\exists_{c}$ are logical constants, but Abstract and Concrete are not. (For it is only the thought that these are standard, non-logical, predicate symbols that justifies the claim that $\alpha_{M}$ is not a logical truth.) However, the work that $\exists_{a}$ and $\exists_{c}$ do in $\mathcal{L}_{P}$ is precisely that which is done by Abstract and Concrete (together with $\exists$ ) in $\mathcal{L}_{M}$. But then, if we are treating the former as logical (as Turner quite naturally is), we should surely also so treat the latter. Indeed, this differential treatment seems particularly

\footnotetext{
${ }^{12}$ There is no comparable example in the opposite direction: i.e. a set $\Gamma \cup\{\varphi\}$ of formulas of $\mathcal{L}_{P}$ such that we similarly seem to have $\Gamma \vDash \varphi \Leftrightarrow t(\Gamma) \vDash t(\varphi)$. But no matter: one counterexample is enough. (Note that we cannot tweak our translation exploiting the fact just mentioned, because the relevant function from $\mathcal{L}_{P}$ to $\mathcal{L}_{M}$ is not surjective.)

${ }^{13}$ I should note that Turner [2012] in fact gives a whole range of arguments aimed at establishing that $\mathcal{L}_{P}$ and $\mathcal{L}_{M}$ are not notational variants. Specifically, aimed at establishing that there is no other translation under which they are such. However, these general arguments make the same assumptions about the logic of $\mathcal{L}_{P}$ and $\mathcal{L}_{M}$ that the particular one does-and that I argue against in $\$ 4$. Thus, if the argument of that section succeeds, then it refutes these general arguments as well.
} 
problematic if one is trying to refute the suggestion that $\mathcal{L}_{P}$ and $\mathcal{L}_{M}$ are notational variants. For the whole thrust of that suggestion is that the relevant expressions are essentially interchangeable. But then, if we are treating one set as logical, we must also treat the other as logical.

To argue as Turner does would seem akin to arguing against the claim that $\mathcal{L}_{\square}$ and $\mathcal{L}_{\diamond}$ are notational variants (under the standard translation), on the basis that if one counts $\square$ as logical, but does not so count $\diamond$, then this translation fails to preserve logic (since one would have $\square F(a) \vDash F(a)$ but $\neg \diamond \neg F(a) \vDash F(a)$, for example). But that would of course be a very unpersuasive way to argue against this claim of notational variance.

The contention that $\exists_{a}$ and $\exists_{c}$, on the one hand, and Abstract and Concrete, on the other, are on a par as far as logicality is concerned can be further supported by considering standard accounts of logical constancy. There is, of course, no universally accepted such account. ${ }^{14}$ But two of the most widely invoked are those in terms of (a) topic neutrality, or (b) permutation invariance. ${ }^{15}$

Consider (a) first: the claim is that an expression is a logical constant iff it can be used to talk about any subject matter. But, of course, the expressions in question are exactly tied in that regard: Abstract can be used to talk about abstract objects completely generally (but not others); exactly what is true of $\exists_{a}$; and similarly for Concrete and $\exists_{c}$.

As for (b), the usual statement of such an account is that an expression $e$ is logical iff for any interpretation $I$ with domain $D$, and any permutation $\pi$ of $D,{ }^{16}$ the semantic value of $e$ in $I, e^{I}$, is unchanged under $\pi$. That is, $\pi\left(e^{I}\right)=e^{I}{ }^{17}$ For example, $=$ passes this test, because $=^{I}=\{\langle d, d\rangle: d \in D\}=\pi\left(=^{I}\right)$ (since $\pi$ is a surjection). Similarly, the test is passed by $\exists$, given that $\exists^{I}=\{X \subseteq D: X \neq \varnothing\}=$ $\{\pi(X): X \subseteq D \wedge X \neq \varnothing\}=\pi\left(\exists^{I}\right) .{ }^{18}$ On the other hand, a standard non-logical unary predicate symbol $F$ will not pass the test: just choose $I$ with $F^{I} \notin\{\varnothing, D\}$, and $\pi$ such that for some $d \in F^{I}, \pi(d) \notin F^{I}$. We then have $\pi\left(F^{I}\right) \neq F^{I}$.

\footnotetext{
${ }^{14}$ See MacFarlane [2015] for a useful survey.

${ }^{15}$ For (a), see Ryle [1954: 111-29] and Peacocke [1976: 229], for (b), Tarski [1986] and McGee [1996].

${ }^{16}$ I.e. any bijection of $D$ into itself.

${ }^{17}$ In the first instance, $\pi$ is defined for members of $D$, but it can be extended to ordered tuples of members of $D$, subsets of $D$ etc. in the obvious way: e.g. if $a, b \in D$, then $\pi(\langle a, b\rangle)=\langle\pi(a), \pi(b)\rangle$; if $X \subseteq D$, then $\pi(X)=\{\pi(d): d \in D\}$; and so on.

${ }^{18}$ As usual, I take the semantic value of a quantifier $Q$ to be a set of subsets of the domain. The idea is that $Q x \varphi$ is true iff the extension of $\varphi$ is in this set.
} 
What happens when we apply this test to the expressions of $\mathcal{L}_{P}$ and $\mathcal{L}_{M}$ ? Consider first $\exists_{a}$ and $\exists_{c}$. In any $\mathcal{L}_{P}$-interpretation $I$, each of these will be assigned its own domain, $D_{a}$ and $D_{c}{ }^{19}$ If $\pi$ is a permutation of the total domain, i.e. $D_{a} \cup D_{c}$, then: $\pi\left(\exists_{a}^{I}\right)=\exists_{a}^{I}$ and $\pi\left(\exists_{c}^{I}\right)=\exists_{c}^{I}$ iff for any $d \in D_{a} \cup D_{c}, d \in D_{a} \Leftrightarrow \pi(d) \in D_{a}$, and $d \in D_{c} \Leftrightarrow \pi(d) \in D_{c}$. That is, the semantic values of $\exists_{a}$ and $\exists_{c}$ are invariant under precisely those permutations that respect the 'abstract'/'concrete' divide: i.e. that send 'abstract' objects of the interpretation to other such objects, and 'concrete' objects to other such ones.

But the situation with Abstract and Concrete is exactly similar. If $I$ is an $\mathcal{L}_{M^{-}}$ interpretation, then it will have a single domain $D$. And a permutation of $D$ will leave Abstract ${ }^{I}$ and Concrete ${ }^{I}$ unchanged iff it similarly respects the 'abstract'/'concrete' divide: i.e. sends 'abstract' members of the domain, which are in this case the members of Abstract $^{I}$, to other such objects, and similarly for 'concrete' ones. Again, then, the two sets of expressions would seem to be precisely tied as far as logicality is concerned.

Thus, given that we are taking $\exists_{a}$ and $\exists_{c}$ to be logical, we should also so take Abstract and Concrete. ${ }^{20}$ We will see, however, that once we do this, our translation $t$ satisfies (LR) after all.

\subsection{Logic for $\mathcal{L}_{P}$ and $\mathcal{L}_{M}$}

I want to show, then, that once we take Abstract and Concrete to be logical, the translation $t$ of $\$ 2$ does indeed preserve logic. As is standard, I assume that logical consequence for a language $\mathcal{L}$ is defined in terms of interpretations of $\mathcal{L}$.

Consider $\mathcal{L}_{P}$ first. The natural notion of an interpretation of $\mathcal{L}_{P}$ is as follows (see Turner [2012: 432]). An $\mathcal{L}_{P}$-interpretation $I$ is a triple $\left\langle D_{a}, D_{c}, i\right\rangle$ such that $D_{a}$ and $D_{c}$ are non-empty ${ }^{21}$ sets (I use $D_{\cup}$ for $D_{a} \cup D_{c}$ ); and $i$ is a function that sends every individual constant of $\mathcal{L}_{P}$ to a member of $D_{\cup}$; every $n$-ary $(n \geq 1)$ function symbol to an $n$-ary function from $D_{\cup}$ to $D_{\cup}$; and every $n$-ary $(n \geq 1)$ predicate symbol to a subset of $D_{\cup}^{n}$. One might also insist that $D_{a}$ and $D_{c}$ are disjoint. And everything that I say would straightforwardly carry over, but for definiteness I assume that $D_{a}$ and $D_{c}$ are allowed to overlap.

An $\mathcal{L}_{P}$-valuation is an $\mathcal{L}_{P}$-interpretation together with an assignment of values to variables. Satisfaction is then defined in the obvious way: with $\exists_{a}$ taken to range

\footnotetext{
${ }^{19}$ As with $\exists, \exists_{a}^{I}=\left\{X \subseteq D_{a}: X \neq \varnothing\right\}$, and similarly for $\exists_{c}$.

${ }^{20}$ What about the possibility of taking none of these expressions to be logical? I consider that towards the end of this section.

${ }^{21}$ We could also allow one or both to be empty, but for simplicity I do not do this.
} 
over $D_{a}$, and $\exists_{c}$ over $D_{c}$. Finally, for a set $\Gamma \cup\{\varphi\}$ of formulas of $\mathcal{L}_{P}$, we say that $\Gamma \vDash \varphi$ if, whenever an $\mathcal{L}_{P}$-valuation satisfies every member of $\Gamma$, it also satisfies $\varphi$.

What, now, about interpretations of $\mathcal{L}_{M}$ ? That is, what is the cash value of the claim that Abstract and Concrete are logical? I suggest that the natural definition of an interpretation of $\mathcal{L}_{M}$ is in fact exactly the same as in the $\mathcal{L}_{P}$ case. It is just that, now, $D_{a}$ is not the range of $\exists_{a}$, but the extension of Abstract (and similarly for $D_{c}$ ). Further, we do not need to supply an additional domain for $\exists$, because this is simply taken to range over $D_{\cup}$.

This would seem to be the natural definition of an $\mathcal{L}_{M}$-interpretation, given that $\exists$ is intended to range over abstract and concrete things-and nothing else. Of course, there are distinct monist languages in which $\exists$ is also intended range over other sorts of thing (e.g. mental items). Such languages will still be notational variants of pluralist ones: but those with additional quantifiers beyond $\exists_{a}$ and $\exists_{c}$ (e.g. $\left.\exists_{m}\right)$.

Here is another way in which one can justify this definition. One aspect of our treating $\exists_{a}$ and $\exists_{c}$ as logical is to insist that the other expressions of the language have their interpretations drawn from the 'abstract' and 'concrete' objects of this interpretation: i.e. the objects that constitute the interpretations of $\exists_{a}$ and $\exists_{c}$, which is to say the members of $D_{\cup}$. Thus, we require that the interpretations of individual constants are members of $D_{\cup}$, the interpretations of $n$-ary predicate symbols are subsets of $D_{\cup}^{n}$, etc. In the case of $=$, for example, this amounts to $=^{I}=\{\langle d, d\rangle: d \epsilon$ $\left.D_{\cup}\right\}$. The natural way of treating Abstract and Concrete as logical would seem to be by imposing a similar requirement. That is, to insist that the interpretations of other expressions be drawn from the objects that constitute those of Abstract and Concrete. In the case of $=$, this again amounts to $=^{I}=\left\{\langle d, d\rangle: d \in D_{\cup}\right\}$. In that of $\exists$, it amounts to $\exists^{I}=\left\{X \subseteq D_{\cup}: X \neq \varnothing\right\}$. But then we have precisely the notion of an $\mathcal{L}_{M}$-interpretation that I have proposed, together with a definition of satisfaction on which $\exists$ ranges over $D_{\cup}$.

We're then home and dry, by the following result (which is proved by a straightforward induction on the degrees of $\varphi$ and $\psi) .{ }^{22}$

Proposition 1. Let $\sigma$ be an $\mathcal{L}_{P}$-valuation (i.e. $\mathcal{L}_{M}$-valuation), $\varphi$ a formula of $\mathcal{L}_{P}$ and $\psi$ one of $\mathcal{L}_{M}$. Then:

(i) $\sigma \vDash \varphi$ iff $\sigma \vDash t(\varphi)$;

\footnotetext{
${ }^{22}$ If $\sigma$ is an $\mathcal{L}_{P}$-valuation, and $\varphi$ is a formula of $\mathcal{L}_{P}$, then $\sigma \vDash \varphi$ means that $\sigma$ satisfies $\varphi$. And similarly for $\mathcal{L}_{M}$. Note that although any $\mathcal{L}_{P}$-valuation is an $\mathcal{L}_{M}$-valuation, and vice versa, ' $\sigma \models$ ' means something rather different, depending on whether we are treating $\sigma$ as an $\mathcal{L}_{P}$-valuation, or an $\mathcal{L}_{M}$ - one. Thus, slightly more verbosely, (i), for example, might be written: $\sigma \vDash_{P} \varphi$ iff $\sigma \vDash_{M} t(\varphi)$.
} 
(ii) $\sigma \vDash \psi$ iff $\sigma \vDash t(\psi)$.

It follows immediately that (LR) is satisfied. For suppose that $\Gamma \cup\{\varphi\}$ is a set of formulas of $\mathcal{L}_{P}$. Then: $\Gamma \vDash \varphi$ iff any $\mathcal{L}_{P}$-valuation satisfying $\Gamma$, satisfies $\varphi$; which, by (i), holds iff any $\mathcal{L}_{M}$-valuation satisfying $t(\Gamma)$, satisfies $t(\varphi)$. Thus, $\Gamma \vDash \varphi$ iff $t(\Gamma) \vDash t(\varphi)$. The case where $\Gamma \cup\{\varphi\}$ is a set of formulas of $\mathcal{L}_{M}$ is exactly similar. As promised, then, (LR) is met after all.

There is one final loose end to tie up. We saw above that if we are taking $\exists_{a}$ and $\exists_{c}$ to be logical, then we should also so take Abstract and Concrete. Just as: if we take $\square$ to be logical, then we should also so take $\diamond$. In the case of $\mathcal{L}_{\square}$ and $\mathcal{L}_{\diamond}$, however, (LR) is satisfied both when we treat $\square$ and $\diamond$ as logical, and when we treat neither as. Might one insist that if $\mathcal{L}_{P}$ and $\mathcal{L}_{M}$ are genuine notational variants, then (LR) must similarly be satisfied both when we treat all of the relevant expressions as logical, and when we treat none of them as?

In fact, unlike in the case of $\mathcal{L}_{\square}$ and $\mathcal{L}_{\diamond}$, (LR) is not satisfied when we treat none of these expressions-i.e. $\exists_{a}, \exists_{c}, \exists$, Abstract and Concrete-as logical. ${ }^{23}$ On reflection, however, to insist that (LR) is satisfied even in this case is unreasonable. This can be seen by considering a simple variant of the modal example: $\mathcal{L}_{\square \diamond}$ and $\mathcal{L}_{\square} \cdot{ }^{24}$ It seems clear that these are notational variants (under the obvious translation $s)$. But (LR) is not satisfied if we treat neither $\square$ nor $\diamond$ as logical: since if $\delta$ is $\neg \square \neg F(a)$ and $\zeta$ is $\diamond F(a)$, then $\delta \not \zeta$; but $s(\zeta)=\delta=s(\delta)$ and so $s(\delta) \vDash s(\zeta)$. It seems, then, that notational variance does not require (LR) to hold even in the case where we treat the expressions being (non-homophonically) translated as nonlogical.

Indeed, what the example of $\mathcal{L}_{\square \diamond}$ and $\mathcal{L}_{\square}$ seems to show is that we can only expect notational variants to satisfy (LR), if we are treating the expressions being translated as logical. A result that lends further-if slightly more indirect-support

\footnotetext{
${ }^{23}$ The following would be a counterexample to (LR). Let $\beta$ be $\exists x(\neg \operatorname{Abstract}(x) \wedge \neg \operatorname{Concrete}(x))$, and $\gamma, \exists x x \neq x$. If we are treating none of the expressions as logical, then $\beta \neq \gamma$. However, the 'double' translation of $\beta, t(t(\beta))$, is

$\exists x(\operatorname{Abstract}(x) \wedge \neg \operatorname{Abstract}(x) \wedge \neg \operatorname{Concrete}(x)) \vee \exists x(\operatorname{Concrete}(x) \wedge \neg \operatorname{Abstract}(x) \wedge$ $\neg \operatorname{Concrete}(x))$,

while $t(t(\gamma))$ is

$\exists x(\operatorname{Abstract}(x) \wedge x \neq x) \vee \exists x(\operatorname{Concrete}(x) \wedge x \neq x)$.

And $t(t(\beta)) \vDash t(t(\gamma))$ (since in each formula $\exists x$ is applied to contradictions).

${ }^{24}$ Of course, these are identical except that while $\mathcal{L}_{\square \diamond}$ contains $\square$ and $\diamond, \mathcal{L}_{\square}$ contains only $\square$.
} 
to the contention above that when we are considering whether $\mathcal{L}_{P}$ and $\mathcal{L}_{M}$ are notational variants, we should treat all of the expressions being translated as logical.

There seems, then, to be every reason to think that $\mathcal{L}_{P}$ and $\mathcal{L}_{M}$ are notational variants, and thus that the debate between the pluralist and the monist is not a substantive one. ${ }^{25}$

\section{Appendix: Sorted Pluralism}

In this appendix, I consider the possibility that the pluralist might propose a sorted language, $\mathcal{K}_{P}$. That is, every term and argument place in $\mathcal{K}_{P}$ has a sort, $a$ or $c$, and a string is a formula only if its terms and argument places match. Again, I assume that the quantifiers of $\mathcal{K}_{P}$ are $\exists_{a}$ and $\exists_{c}$. In this case, too, there is a monist language that seems to be a notational variant of the pluralist one.

As in the unsorted case, the main choice point in the logic of $\mathcal{K}_{P}$ is whether to allow the domains of $\exists_{a}$ and $\exists_{c}$ to overlap. Here it seems most natural not to allow this: the idea of a sorted language goes naturally with that to the effect that we are dealing with two fundamentally different, and thus mutually exclusive, types of things. Thus, that is the approach that I consider. But one could easily enough derive similar conclusions on the overlap approach.

A $\mathcal{K}_{P}$-interpretation is just like an $\mathcal{L}_{P^{-}}$one, except that sorts are respected. That is, if $I=\left\langle D_{a}, D_{c}, i\right\rangle$ is a $\mathcal{K}_{P}$-interpretation, and $b_{a}$ is an individual constant of sort $a$, then $i\left(b_{a}\right) \in D_{a}$; and if $R_{a c}$ is a binary predicate symbol of sort $\langle a, c\rangle$ (the first argument place is of sort $a$, the second of sort $c$ ), then $i\left(R_{a c}\right) \subseteq D_{a} \times D_{c}$; and so on.

Let $\mathcal{K}_{M}$ be just like $\mathcal{K}_{P}$, except that it contains Abstract $_{a}$, Concrete ${ }_{c}$ and $\exists$ in place of $\exists_{a}$ and $\exists_{c}$, and variables are no longer sorted. Thus, e.g., $R_{a c}(x, x)$ is a formula of $\mathcal{K}_{M}$. In $\mathcal{K}_{M}$ individual constants and argument places are sorted: this is required if even the quantifier- and variable-free fragment of the language is to be translatable into $\mathcal{K}_{P}$. However, the machinery of quantification-the quantifier and variables-is unsorted, and so it is in this sense a genuinely monist language. It will follow from that fact that $\mathcal{K}_{M}$ and $\mathcal{K}_{P}$ are notational variants that, given this quantifier- and variable-free base, the choice of whether to quantify in a monist or a pluralist fashion is not a substantive one.

As in the unsorted case, a $\mathcal{K}_{M}$-interpretation is simply a $\mathcal{K}_{P}$-interpretation.

\footnotetext{
${ }^{25}$ [Acknowledgements.]
} 
How can we translate between these languages? To keep things simple, I give a translation only for sentences: this satisfies (LR) in the sense that the logic of sentences is preserved. If one thinks that true notational variance requires a translation that works for formulas more generally, then one could extend that which I give, but for reasons of space I do not do this here.

The translation $s$ is defined as follows. From $\mathcal{K}_{P}$ to $\mathcal{K}_{M}$ this works just as before (i.e. like $t$ of $\$ 2$ ). The opposite direction is slightly more involved, given the fact that variables are sorted in $\mathcal{K}_{P}$ but not in $\mathcal{K}_{M}$ : if we tried to translate as before, we would take some formulas of $\mathcal{K}_{M}$ to illformed strings.

Instead, I define a function $s_{\mathrm{O}}$ from formulas of $\mathcal{K}_{M}$ to strings of $\mathcal{K}_{P}$ as follows. If $\varphi$ is $\operatorname{Abstract}_{a}(r)$, then $s_{\mathrm{o}}(\varphi)$ is $\exists_{a} x_{a} x_{a}={ }_{a a} r$; if $\varphi$ is $\operatorname{Concrete}_{c}(r)$, then $s_{\mathrm{o}}(\varphi)$ is $\exists_{c} x_{c} x_{c}=_{c c} r$; if $\varphi$ is atomic of some other form, then $s_{0}(\varphi)=\varphi$; if $\varphi$ is $\neg \psi$, then $s_{0}(\varphi)=\neg s_{0}(\psi)$; if $\varphi$ is $\psi \wedge \chi$, then $s_{0}(\varphi)=s_{0}(\psi) \wedge s_{0}(\chi)$; and if $\varphi$ is $\exists x \psi$, then $s_{0}(\varphi)=\exists_{a} x_{a}\left[s_{0}(\psi)\right]_{a / x} \vee \exists_{c} x_{c}\left[s_{0}(\psi)\right]_{c / x}$, where $\chi_{a / x}$ is the result of replacing every occurrence of an atomic formula with $x$ in an argument place of sort $c$ with $\perp$, and then replacing every remaining occurrence of $x$ with $x_{a}$ (and similarly for $\chi_{c / x}$ ).

It is then easy to see that if $\beta$ is a sentence of $\mathcal{K}_{M}, s_{0}(\beta)$ is one of $\mathcal{K}_{P}$. We thus set $s(\beta)=s_{0}(\beta)$. Although for reasons of space I will not make the case in any detail, it is at least very plausible that $s$ preserves both content (in the sense of $\$_{1}$ ) and metaphysical perspicuity, and thus that $\mathcal{K}_{P}$ and $\mathcal{K}_{M}$ are notational variants under $s$. Further, we have the following, which ensures that (LR) is indeed satisfied for sentences.

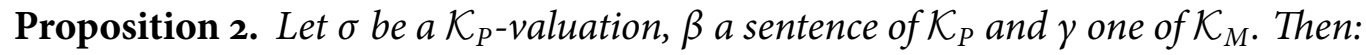

(i) $\sigma \vDash \beta$ iff $\sigma \vDash s(\beta)$;

(ii) $\sigma \vDash \gamma$ iff $\sigma \vDash s(\gamma)$.

\section{References}

Caplan, B. 2011. Ontological Superpluralism. Philosophical Perspectives 25: 79-114.

Frege, G. 1884. The Foundations of Arithmetic: A Logico-Mathematical Enquiry into the Concept of Number. Translated by J. L. Austin (1953). Oxford: Basil Blackwell.

-1891. Function and Concept. In P. Geach and M. Black (eds), Translations from the Philosophical Writings of Gottlob Frege (1980): 21-41. Oxford: Basil Blackwell. 
-1892. On Concept and Object. In P. Geach and M. Black (eds), Translations from the Philosophical Writings of Gottlob Frege (1980): 42-55. Oxford: Basil Blackwell.

Hale, B. and C. Wright. 2001. The Reason's Proper Study: Essays towards a NeoFregean Philosophy of Mathematics. Oxford: Clarendon Press.

Lewis, D. 1983. New Work for a Theory of Universals. Australasian Journal of Philosophy 61: 343-77.

-1986. On the Plurality of Worlds. Oxford: Blackwell Publishing.

MacFarlane, J. 2015. Logical Constants. In E. N. Zalta (ed.), The Stanford Encyclopedia of Philosophy (Fall 2015 Edition). URL $=<$ http://plato.stanford.edu/archives/fall2015/entries/logical-constants/>.

McDaniel, K. 2009. Ways of Being. In D. Chalmers, D. Manley and R. Wasserman (eds), Metametaphysics: 290-319. Oxford University Press.

-2010a. Being and Almost Nothingness. Noûs 44: 628-49.

-2010b. A Return to the Analogy of Being. Philosophy and Phenomenological Research 81: 688-717.

McGee, V. 1996. Logical Operations. Journal of Philosophical Logic 25: 567-80.

Peacocke, C. 1976. What is a Logical Constant? Journal of Philosophy 73: 221-40.

Prior, A. N. 1971. Objects of Thought. P. T. Geach and A. J. P. Kenny (eds). Oxford: Clarendon Press.

Russell, B. 1908. Mathematical Logic as Based on the Theory of Types. American Journal of Mathematics 30: 222-62.

Ryle, G. 1954. Dilemmas. Cambridge University Press.

Sider, T. 2011. Writing the Book of the World. Oxford University Press.

Spencer, J. 2012. Ways of Being. Philosophy Compass 7: 910-18

Tarski, A. 1986. What are Logical Notions? History and Philosophy of Logic 7: 14354.

Turner, J. 2010. Ontological Pluralism. Journal of Philosophy 107: 5-34.

-2012. Logic and Ontological Pluralism. Journal of Philosophical Logic 41: 419-48.

Willamson, T. 2003. Everything. Philosophical Perspectives 17: 415-65. 